\title{
Metabolic engineering of Escherichia coli for high-specificity production of isoprenol and prenol as next generation of biofuels
}

Yanning Zheng ${ }^{1,2}$, Qiang Liư ${ }^{3}$, Lingling Li ${ }^{3}$, Wen Qin ${ }^{3}$, Jianming Yang ${ }^{1}$, Haibo Zhang ${ }^{1}$, Xinglin Jiang ${ }^{1,2}$, Tao Cheng ${ }^{1}$, Wei Liu ${ }^{1}$, Xin Xu ${ }^{1}$ and Mo Xian ${ }^{1 *}$

\begin{abstract}
Background: The isopentenols, including isoprenol and prenol, are excellent alternative fuels. However, they are not compounds largely accumulated in natural organism. The need for the next generation of biofuels with better physical and chemical properties impels us to develop biosynthetic routes for the production of isoprenol and prenol from renewable sugar. In this study, we use the heterogenous mevalonate-dependent (MVA) isoprenoid pathway for the synthesis of isopentenyl pyrophosphate (IPP) and dimethylallyl pyrophosphate (DMAPP) intermediates, and then convert IPP and DMAPP to isoprenol and prenol, respectively.

Results: A mevalonate titer of $1.7 \mathrm{~g} / \mathrm{L}$ was obtained by constructing an efficient MVA upper pathway in engineered E. coli. Different phosphatases and pyrophosphatases were investigated for their abilities in hydrolyzing the IPP and DMAPP. Consequently, ADP-ribose pyrophosphatase was found to be an efficient IPP and DMAPP hydrolase. Moreover, ADP-ribose pyrophosphatase from Bacillus subtilis (BsNudF) exhibited a equivalent substrate specificity towards IPP and DMAPP, while ADP-ribose pyrophosphatase from E. coli (EcNudF) presented a high substrate preference for DMAPP. Without the expression of any phosphatases or pyrophosphatases, a background level of isopentenols was synthesized. When the endogenous pyrophosphatase genes (EcNudF and yggV) that were capable of enhancing the hydrolyzation of the IPP and DMAPP were knocked out, the background level of isopentenols was still obtained. Maybe the synthesized IPP and DMAPP were hydrolyzed by some unknown hydrolases of E. coli. Finally, $1.3 \mathrm{~g} / \mathrm{L}$ single isoprenol was obtained by blocking the conversion of IPP to DMAPP and employing the BsNudF, and $0.2 \mathrm{~g} / \mathrm{L} \sim 80 \%$ prenol was produced by employing the EcNudF. A maximal yield of $12 \%$ was achieved in both isoprenol and prenol producing strains.

Conclusions: To the best of our knowledge, this is the first successful report on high-specificity production of isoprenol and prenol by microbial fermentation. Over $1.3 \mathrm{~g} / \mathrm{L}$ isoprenol achieved in shake-flask experiments represents a quite encouraging titer of higher alcohols. In addition, the substrate specificities of ADP-ribose pyrophosphatases were determined and successfully applied for the high-specificity synthesis of isoprenol and prenol. Altogether, this work presents a promising strategy for high-specificity production of two excellent biofuels, isoprenol and prenol.
\end{abstract}

Keywords: Isoprenol, Prenol, Metabolic engineering, Escherichia coli, Biofuel

\footnotetext{
*Correspondence: xianmo@qibebt.ac.cn

${ }^{1}$ CAS Key Laboratory of Biobased Materials, Qingdao Institute of Bioenergy and Bioprocess Technology, Chinese Academy of Sciences, No.189 Songling Road, Laoshan District, Qingdao 266101, China

Full list of author information is available at the end of the article
} 


\section{Background}

With increasing concerns about environmental problems and energy security, research interest has been aroused in the field of microbial production of fuels and chemicals from renewable sources [1-4]. Short chain alcohols (C2C5) represent a category of primary biofuels. Compared with the ethanol (C2), a main biofuel in use today [5], the C5 alcohols have higher energy density and lower water miscibility [1,6]. Moreover, the branched-chain unsaturated isopentenols (isoprenol and prenol) possess higher octane numbers and better low-temperature fluidity than their straight-chain saturated counterpart. In addition, by removing a molecule of water, isoprenol can be converted to isoprene, a very important monomer for synthesizing rubber. Global rubber demand is forecast to reach 27.2 million metric tons in 2012. Prenol is also of great importance as a building block to synthesize pyrethroid, a widely used pesticide. However, the isoprenol and prenol, which are conventionally manufactured from petroleum-derived isobutene and formaldehyde, are not compounds largely accumulated in natural organism. The need for the next generation of biofuels with better physical and chemical properties impels us to seek a biological route for direct production of isopentenols from renewable sugar in a microbial fermentation process.

To obtain the $\mathrm{C} 5$ backbones, we paid attention to the isoprenoid biosynthetic pathways, which exist as mevalonatedependent (MVA) isoprenoid pathway in eukaryotes or deoxyxylulose 5-phosphate (DXP) pathway in plants and most prokaryotes [7]. The MVA and DXP pathways both end with the synthesis of isopentenyl pyrophosphate (IPP) and dimethylallyl pyrophosphate (DMAPP), which are used as the $\mathrm{C} 5$ building blocks for preparing isoprenoids in vivo [7-10]. Expression of the heterologous MVA pathway can avoid the unknown physiological control elements in E. coli and thus result in a high-level production of isoprenoid precursors [7]. Therefore, we took advantage of the MVA pathways for enhanced production of the precursors, IPP and DMAPP. The intermediates IPP and DMAPP can be finally converted to isoprenol and prenol by removing their pyrophosphates (Figure 1).

The recovery of the desired products will be greatly simplified if the single isoprenol or prenol can be harvested in the culture. However, thus far, no study has been carried out for high-specificity production of isoprenol and prenol in either wild-type or engineered microbes. To reach this goal, firstly, it is needed to employ phosphatases or pyrophosphatases with appropriate substrate specificities. Secondly, it is also needed to adjust the carbon flux into IPP and DMAPP, which can be interconverted with the help of isopentenyl pyrophosphate isomerase [11]. But unfortunately, no such efforts were made to tailor the composition of the isopentenols.

In this study, we devised a biosynthetic approach to selectively produce isoprenol and prenol in engineered E. coli. To improve the production of isoprenol and prenol, we compared the efficacies of different phosphatases and pyrophosphatases, and selectively directed the

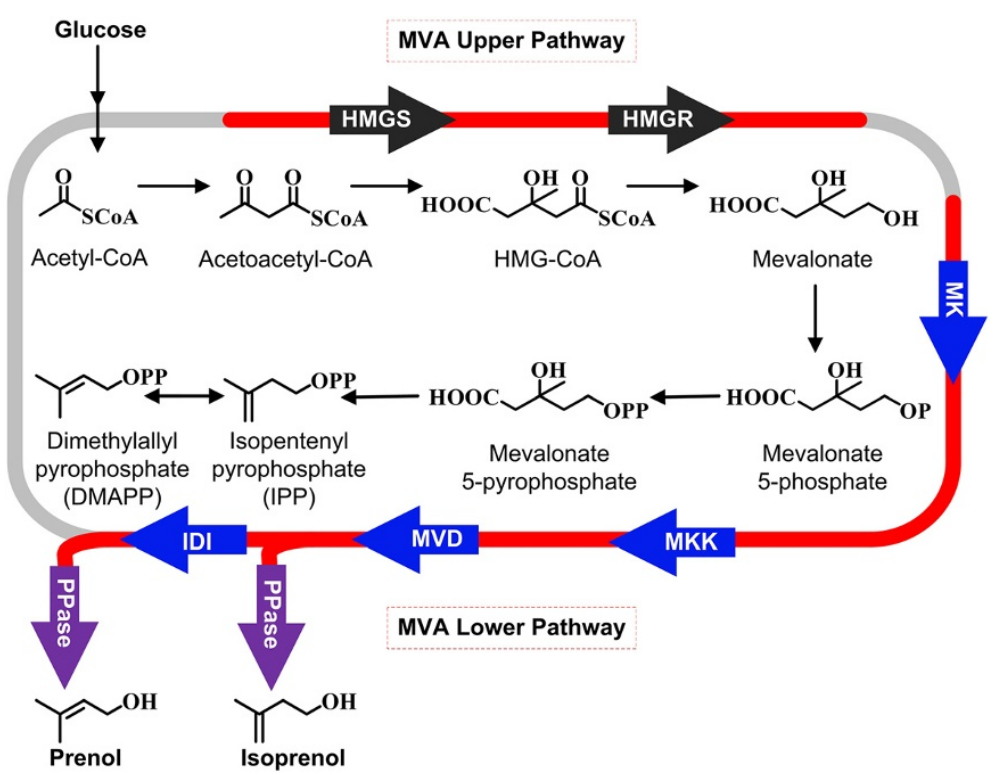

Figure 1 Isoprenol and prenol biosynthesis pathway. The heterogenous pathway that was introduced into $E$. coli is indicated by the red line. The black arrow represents the MVA upper pathway, the blue arrow represents the MVA lower pathway, and the purple arrow represents the isopentenol forming pathway. HMG-CoA, 3-hydroxy-3-methylglutaryl-coenzyme A; HMGS, HMG-CoA synthase; HMGR, HMG-CoA reductase; MK, mevalonate kinase; MKK, phosphomevalonate kinase; MVD, mevalonate pyrophosphate decarboxylase; IDI, isopentenyl pyrophosphate: dimethylallyl pyrophosphate isomerase. 
metabolic flux into the isoprenol versus prenol by inactivation of the heterogenous isopentenyl pyrophosphate isomerase or overexpression of a selective pyrophosphatase. We also discussed the effect of different pyrophosphatases on the hydrolyzation of IPP and DMAPP.

\section{Results}

Enhanced production of mevalonate

The MVA pathway can be divided into upper pathway and lower pathway, which are connected by mevalonate (Figure 1). To convert glucose to mevalonate, it is needed to introduce the exogenous HMG-CoA synthase and HMG-CoA reductase into the $E$. coli cell. We first expressed the HMG-CoA synthase (HMGS) and the truncated HMG-CoA reductase ( $\mathrm{t} H M G R$ ) from Saccharomyces cerevisiae [12], but the resultant engineered strain only produced $36.7 \mathrm{mg} / \mathrm{L}$ mevalonate. Tabata and Hashimoto improved the production of mevalonate by expressing a mevalonate pathway derived from Enterococcus faecalis [13]. To further enhance the synthesis of mevalonate in our study, we next employed the mvaS and mvaE from E. faecalis as the HMG-CoA synthase and HMG-CoA reductase, respectively. The yielded strain had a 45 -fold increase in mevalonate accumulation, reaching a titer of $1.7 \mathrm{~g} / \mathrm{L}$. The improved mevalonate production may be attributed to the additional acetyl-CoA acetyltransferase activity of MvaE, which can promote the conversion of acetyl-CoA to acetoacetyl-CoA [14].

\section{High-specificity production of isoprenol and prenol}

The conversion of mevalonate to IPP and DMAPP requires four steps, sequentially catalyzed by mevalonate kinase, phosphomevalonate kinase, mevalonate pyrophosphate decarboxylase and isopentenyl pyrophosphate: dimethylallyl pyrophosphate isomerase, whose coding genes in S. cerevisiae S288c are ERG12, ERG8, ERG19 and IDI1, respectively [15-18]. We cloned the four genes of $S$. cerevisiae into the pTrcHis2B to generate pTrcLower expression vector, with a method of successive hybridization [19]. Expression of the whole MVA pathway in E. coli (control strain) resulted in $4.6 \mathrm{mg} / \mathrm{L}$ isoprenol and $16.2 \mathrm{mg} / \mathrm{L}$ prenol (Figure 2a, Control).

The low level of isopentenol production is probably attributed to the poor phosphatase or pyrophosphatase activity in $E$. coli. Therefore, it is needed to overexpress phosphatase or pyrophosphatase that can efficiently hydrolyze the phosphoester bond of IPP or DMAPP. Phosphatases DPP1 and LPP1 from $S$. cerevisiae were found to be able to promote the production of geranylgeraniol by removing the pyrophosphatases of geranylgeranyl diphosphate [20]. The alkaline phosphatase is a nonspecific phosphomonoesterase that can remove the inorganic phosphate to release corresponding alcohols [21]. It is possible that these phosphatases also have catalytic activities towards IPP and DMAPP. So we tested the efficiencies of the phosphatases 'PhoA (a leaderless version of PhoA) from E. coli, 'DPP1 (a leaderless version of DPP1)

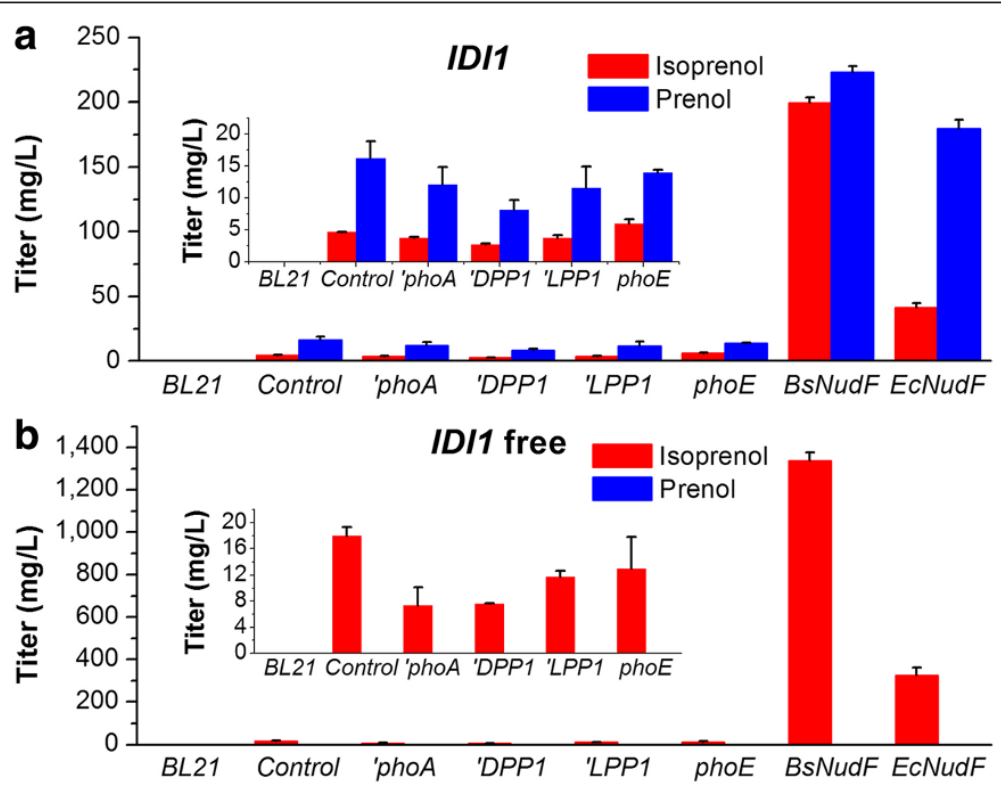

Figure 2 Isoprenol and prenol production by employing different phosphatases and pyrophosphatases. a, strains with the expression of S. cerevisiae isopentenyl pyrophosphate:dimethylallyl pyrophosphate isomerase gene IDI1; $\mathbf{b}$, strains without the expression of IDI1. BL21, the wildtype E. coli BL21(DE3); Control, without the overexpression of any phosphatase or pyrophosphatase gene; 'phoA, phosphatase gene 'phoA from $E$. coli; 'DPP1 and 'LPP1, phosphatase genes 'DPP1 and 'LPP1 from S. cerevisiae; phoE, phosphatase gene from B. subtilis; BsNudF, the ADP-ribose pyrophosphatase gene from B. subtilis; EcNudF, the ADP-ribose pyrophosphatase gene from E. coli. The error bars represent the range from three independent experiments. 
from $S$. cerevisiae, 'LPP1 (a leaderless version of LPP1) from S. cerevisiae and PhoE from B. subtilis on the production of isopentenols. The engineered strains employing the four phosphatases still only produced the background level of isopentenols (Figure 2, 'phoA, 'DPP1, 'LPP1 and phoE), suggesting that these phosphatases can not efficiently remove the pyrophosphates of IPP and DMAPP.

So we further introduced the ADP-ribose pyrophosphatase gene from B. subtilis (BsNudF) into the strain that employed the whole MVA pathway [22]. The resultant $B s N u d F$ expressing strain dramatically enhanced the synthesis of isoprenol and prenol (Figure 2a, BsNudF). This result demonstrates that the ADP-ribose pyrophosphatase is a good candidate biocatalyst to remove the pyrophosphate from IPP and DMAPP.

We noticed that expression of $B s N u d F$ with the whole MVA pathway (YY158) yielded a blend of isoprenol and prenol in the ratio of $\sim 1: 1$ (Figure 2a, $B s N u d F$ ), suggesting that BsNudF has high affinity for both IPP and DMAPP. The recovery of the desired products will be greatly simplified if the single isoprenol or prenol can be harvested in the culture. It is needed for us to tailor the composition of the products by further metabolic engineering. To obtain a single isoprenol, we blocked the conversion of IPP to DMAPP by deleting the IDI1 gene of the pTrcLower. Indeed, the strains without the expression of IDI1 synthesized the isoprenol as their single product (Figure 2b). Finally, an engineered strain expressing $B s N u d F$ and partial MVA pathway (YY159) produced 1,337 mg/L isoprenol in shake flask, with prenol nearly undetected (Figure 2b, $B s N u d F$; Figure 3). This result demonstrates that the metabolic flux of IPP to DMAPP was successfully blocked, and nearly all synthesized IPP entered the isoprenol biosynthetic pathway, contributing to the greatly improved isoprenol production.

To raise the specificity for prenol production, it is needed to find a pyrophosphatase that has a higher substrate preference towards DMAPP. As above mentioned, the strain without expression of any phosphatase and pyrophosphatase produced $20.8 \mathrm{mg} / \mathrm{L}$ isoprenol and prenol, with the main contribution coming from prenol (Figure 2a, Control). This result suggests some endogenous pyrophosphatase of $E$. coli probably contributed to the background level of isopentenols, given the expression of phosphatase didn't exhibit any enhanced production of isopentenols. In addition, expression of ADP-ribose pyrophosphatase from B. subtilis (BsNudF) greatly enhanced the production of isopentenols as aforementioned. Therefore, we paid attention to the ADPribose pyrophosphatase of E. coli (encoded by EcNudF gene). By overexpressing the $E c N u d F$ and the entire MVA pathway (YY168), $0.2 \mathrm{~g} / \mathrm{L}$ isopentenols were finally achieved, with prenol accounting for over $80 \%$ (Figure 2a, EcNudF; Figure 3).

\section{Knockout of the endogenous pyrophosphatase genes of E. coli}

As above mentioned, isopentenols can be synthesized in small amounts even if no pyrophosphatase was overexpressed. We speculated the ADP-ribose pyrophosphatases of E. coli (EcNudF) contributed to the background level of isopentenols, given the overexpression of $E c N u d F$ notably enhanced the production of isopentenols. However, when the endogenous $E c N u d F$ gene of $E$. coli was knocked out, the background level of isopentenols was still observed.

To test if other pyrophosphatases are also capable of hydrolyzing the IPP and DMAPP, we overexpressed E. coli NADH pyrophosphatase (nudC), dITP/XTP pyrophosphatase $(y g g V)$, UDP-2,3-diacylglucosamine pyrophosphatase $(l p x H)$, phosphoribosyl-ATP pyrophosphatase (hisl), inorganic pyrophosphatase (ppa) and CDP-diacylglycerol pyrophosphatase $(c d h)$ in ISP2148 strain, respectively. Overexpression of $y g g V$ improved the isopentenols to some extent, while overexpression of other pyrophosphatases did not enhance the production of isopentenols (Figure 4). It demonstrates that dITP/XTP pyrophosphatase is also able to hydrolyze the pyrophosphates of IPP and DMAPP.

We further deleted the $y g g V$ gene in the $E c N u d F$ gene knockout mutant, yielding a $E c N u d F-y g g V$ double-gene knockout mutant. However, expression of the MVA pathway in this double-gene knockout mutant still synthesized a background level of isopentenols. To check if the background level of isopentenols was produced by the spontaneous hydrolysis of IPP and DMAPP, we determined the IPP and DMAPP hydrolysis in an enzymefree solution. However, no isopentenols was formed after incubating the solution for the same time as the shakeflask experiment. Therefore, it is speculated that some unknown $E$. coli hydrolases resulted in the background level of isopentenols.

\section{Effect of mevalonate accumulation on the production of isopentenols}

It has been known that mevalonate is an important intermediate in the isopentenol biosynthetic pathways. The isopentenol production may be further improved by accelerating the synthesis of mevalonate. It was found that the native HMG-CoA synthase MvaS had a non-optimal catalytic efficiency. When the Ala-110 of the MvaS was replaced by glycine, the mutated enzyme MvaSA110G achieved a 140-fold increase in overall reaction rate [23]. In addition, the biosynthesis of isoprenoid compounds was found to be proportional to the amount of mevalonate, up to a concentration of over $5 \mathrm{~g} / \mathrm{L}$ mevalonate [7].

Therefore, we substituted the MvaS with the MvaSA110G as the HMG-CoA synthase to further improve the production of mevalonate. But unexpectedly, the strain harboring 


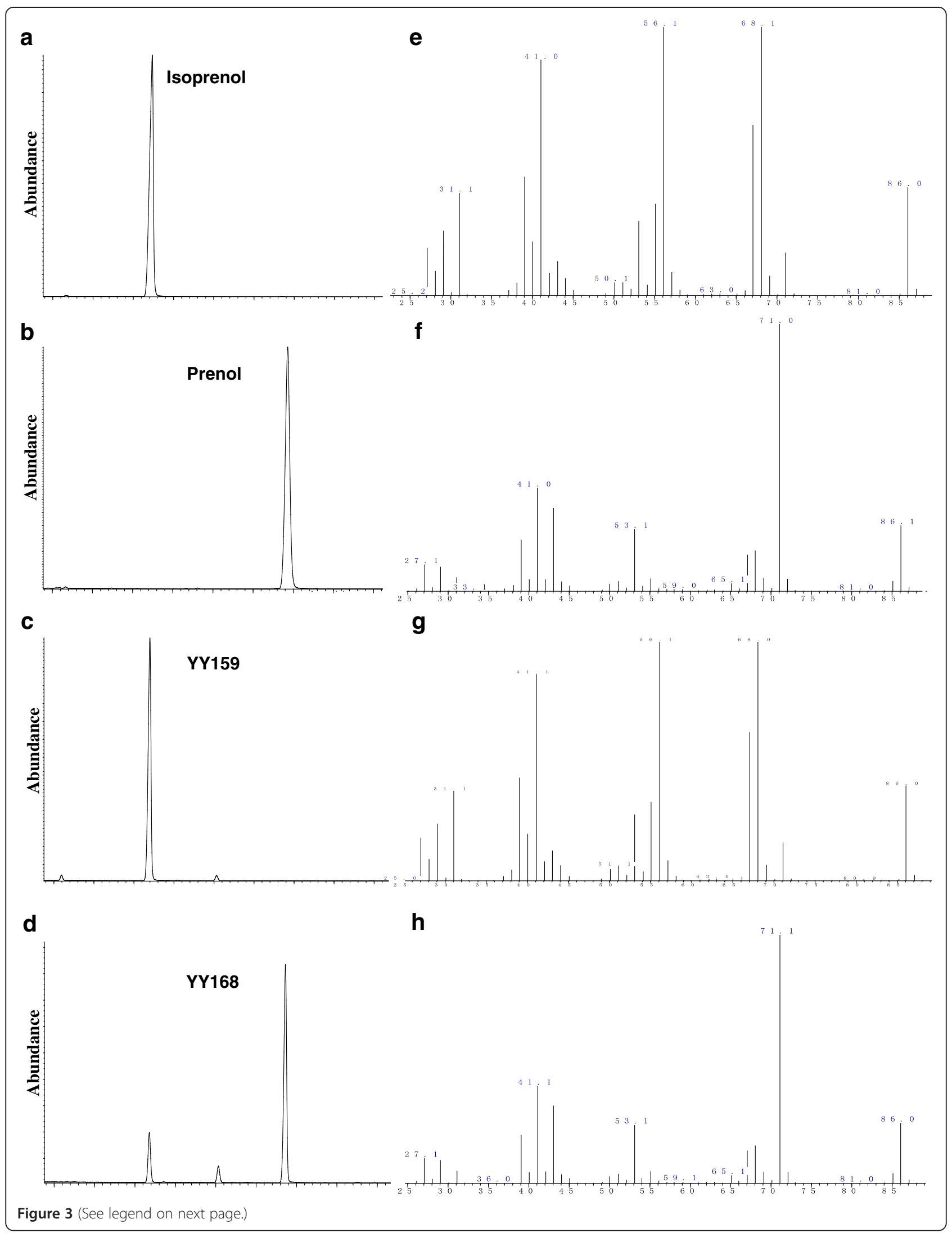


(See figure on previous page.)

Figure 3 GC-MS analysis of isoprenol and prenol in the cultures. By comparing with the authoritative isoprenol (a, e) and prenol (b, $\mathbf{f})$, the capacities of isoprenol and prenol biosynthesis were verified in the engineered strains YY159 and YY168, respectively. YY159 produced the isoprenol as its single product $(\mathbf{c}, \mathbf{g})$, while $Y Y 168$ produced a blend of isoprenol and prenol, with prenol as the dominant component (d, h). $\mathbf{a}$, b, c, d, total ion chromatogram (TIC); e, $\mathbf{f}, \mathbf{g}, \mathbf{h}$, mass spectrum.

the MvaSA110G (ISP214m) only produced $1.23 \mathrm{~g} / \mathrm{L}$ mevalonate, decreased by $25.5 \%$ when compared with the strain carrying MvaS (ISP214) (Figure 5a). The reduced supply of mevalonate directly led to the decreased output of isoprenol and prenol. The isoprenol and prenol production decreased to $0.7 \mathrm{~g} / \mathrm{L}(\mathrm{YY} 159 \mathrm{~m})$ and $0.08 \mathrm{~g} / \mathrm{L}$ (YY168m), respectively (Figure 5b; 5c). It is surprising that the accelerated reaction rate of MvaS did not enhance the production of both mevalonate and isopentenols. A future metabolic flux analysis will make this conversion process clearer.

\section{Evaluation of the isopentenol production of two optimized strains}

Thus far, YY159 and YY168 are the best-performed strains for the production of isoprenol and prenol, respectively. To better understand their isopentenol production, we determined their product titers and yields in specified periods of time. YY159 and YY168 shared a similar process in the production of isopentenols. They both reached their highest isopentenol titers approximately $32 \mathrm{~h}$ post-induction (Figure $6 \mathrm{a} ; 6 \mathrm{~b}$ ). The linear isoprenol productivity of YY159 and prenol productivity of YY168 appeared approximately $4 \sim 8 \mathrm{~h}$ post-induction, constant at $59.3 \mathrm{mg} / \mathrm{L} / \mathrm{h}$ and $9.2 \mathrm{mg} / \mathrm{L} / \mathrm{h}$, respectively. In

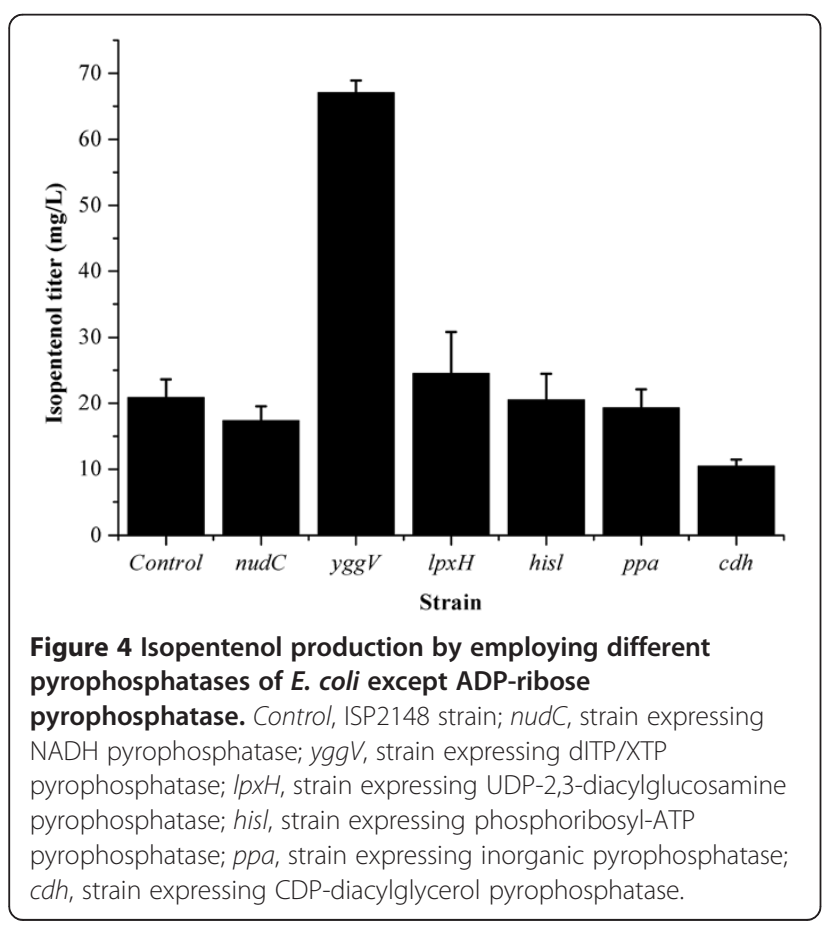

addition, the maximal isopentenol yield of $12 \%$ (i.e. $0.12 \mathrm{~g}$ isopentenols/g glucose consumed) was observed in both YY159 and YY168 (Figure 6c; 6d).

\section{Discussion}

In this study, we present a microbial system for highspecificity production of isoprenol and prenol, two promising new generation of biofuels, from sugar feedstocks. It mainly employs the MVA pathway that is used for isoprenoid biosynthesis in nature $[7,8]$, and finally directs the IPP or DMAPP intermediate to pyrophosphate hydrolization pathway for the production of isoprenol or prenol. This endeavor met with several major challenges: the construction of an efficient MVA pathway to IPP and DMAPP from central metabolic intermediates, the exploration of phosphatases or pyrophosphatases with appropriate substrate specificities, and the engineering of the host $E$. coli metabolism to selectively channel carbon flux and energy into isoprenol or prenol biosynthetic pathway. The challenges met here are likely to be characteristic for high-specificity production of any isomers or homologues biologically, as these compounds are quite similar in structure. Our provided approaches to these problems may contribute to biocatalytic production of more challenging relevant targets.

Our success in respectively producing over $1.3 \mathrm{~g} / \mathrm{L}$ single isoprenol and $0.2 \mathrm{~g} / \mathrm{L} \sim 80 \%$ prenol from two engineered $E$. coli strains demonstrates this strategy is promising for the production of next generation of biofuels. Though it is still needed to further improve the production of the isoprenol and prenol by detailed optimization of the isopentenol biosynthetic pathways, our results are encouraging from several standpoints.

First, we achieved the production of isoprenol and prenol with high specificity. To our knowledge, this is the first successful report on high-specificity production of isoprenol and prenol from renewable sugar.

Second, we constructed and expressed an efficient isopentenol biosynthetic pathway. Over $1.3 \mathrm{~g} / \mathrm{L}$ isoprenol achieved in lab-scale shake-flask experiments is a quite encouraging isopentenol titer, demonstrating that this strategy is promising for the commercial production of higher-alcohol biofuels.

Third, we found that the ADP-ribose pyrophosphatase from different organism had varied substrate preferences towards IPP and DMAPP. In earlier study, no attention was paid to the substrate specificity of ADP-ribose pyrophosphatase. Our finding opens the door to tailoring 

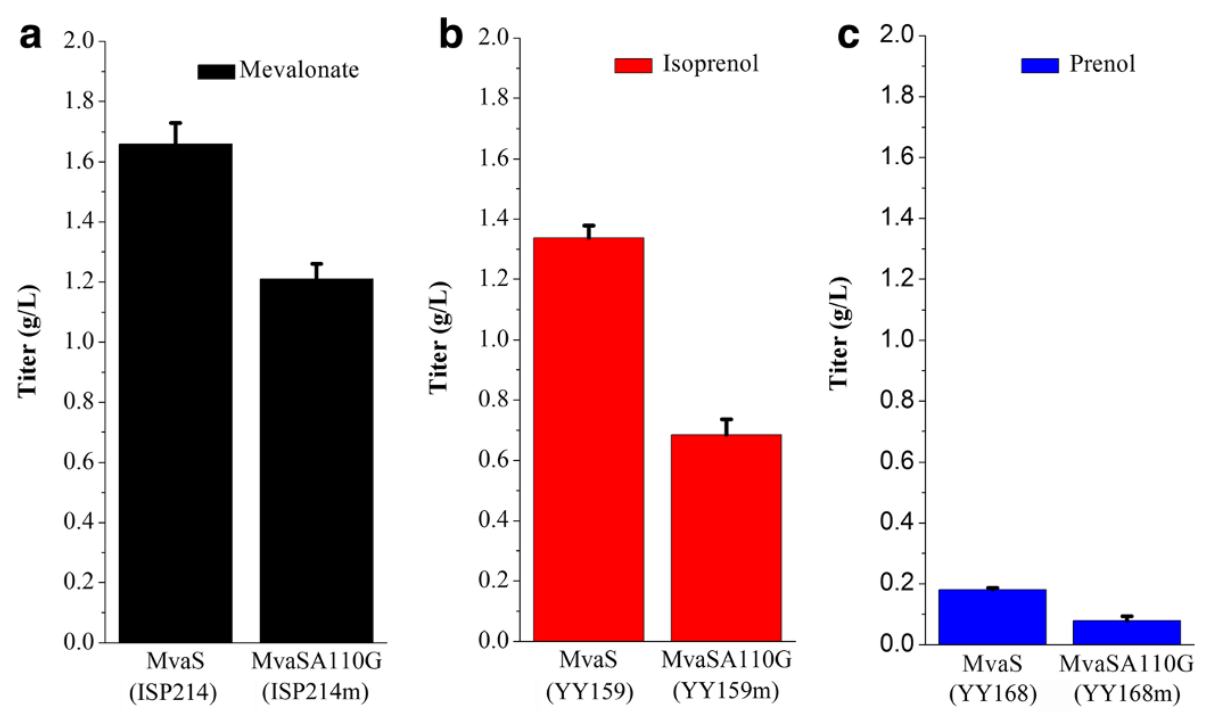

Figure 5 The effect of a mutated HMG-CoA synthase (MvaSA110G) on the production of mevalonate, isoprenol and prenol. The strain employing MvaSA110G (ISP214m) produced a lower titer of mevalonate than the strain carrying the native enzyme MvaS (ISP214) (a). And in the meanwhile, the decreased production of isoprenol (b) or prenol (c) was respectively obtained in two strains expressing the MvaSA110G (YY159 and YY168). The error bars represent the range from two independent experiments.

the composition of isopentenols by employing different pyrophosphatase. Developing pyrophosphatase with higher hydrolyzing efficiency and specificity may contribute to a further improvement of the isopentenol production.

Fourth, the yield of $12 \%$ achieved in shake flask was observed during the post-induction, linear isopentenol production period. This is a rather high yield, even slightly higher than the well developed 3-methyl-1-butanol production [6]. A further yield improvement will be obtained under controlled conditions by using a scalable fermentation system.

Our data also suggests that mevalonate is an important metabolite in determining the production of isoprenol, given that over $80 \%$ mevalonate can be converted to isoprenol. It provides another clue to further increase the production of isoprenol.

Compared with the isoprenol production, a much lower titer of prenol was obtained. The lower biomass probably resulted in the decreased prenol production, given the prenol producing strain (YY168) did not grow so well as the strain for isoprenol synthesis (YY159). Though YY168 finally obtained a higher biomass than YY159, YY168 had lower biomasses during the period when the isopentenols were largely produced (Figure 6). To check if the products prenol and isoprenol inhibit the normal growth of $E$. coli, we measured the growth of E. coli BL21(DE3) in media supplemented with increasing concentrations of exogenous prenol or isoprenol. A slight growth inhibition was indeed observed in the presence of prenol, but the inhibitory effect of prenol on the growth of $E$. coli was even weaker than that of isoprenol (Figure 7). Moreover, the strain YY168 produced a much lower product titer than YY159, which grew well during the production of isoprenol. Therefore, the growth inhibition of strain YY168 was not caused by the synthesized prenol.

Martin et al. found that, using mevalonate as substrate, the expression of partial MVA lower pathway (MK, MKK and MVD) resulted in the highest concentration of IPP, the expression of entire MVA lower pathway (MK, MKK, MVD and IDI) led to a decreased concentration of IPP, and the expression of an additional synthetic pathway that converts IPP and DMAPP to other metabolites further decreased the intracellular concentration of IPP [7]. That is to say, IPP will largely accumulate in engineered $E$. coli if it can't be efficiently converted to other metabolites. In the meanwhile, compared to BsNudF, EcNudF has a much poorer substrate specificity towards IPP, making IPP can't be efficiently hydrolyzed. Therefore, the lower cell density of YY168 may be attributed to the accumulation of IPP, which is toxic and can inhibit normal cell growth [7].

\section{Conclusions}

The engineering strategy described above inaugurates a new realm for the production of alcoholic biofuels. The substrate specificities of ADP-ribose pyrophosphatases were determined and successfully applied for the highspecificity synthesis of isoprenol and prenol. The desired isoprenol and prenol were finally produced with high specificities, being the first successful report on high-specificity production of isoprenol and prenol from renewable sugar. 

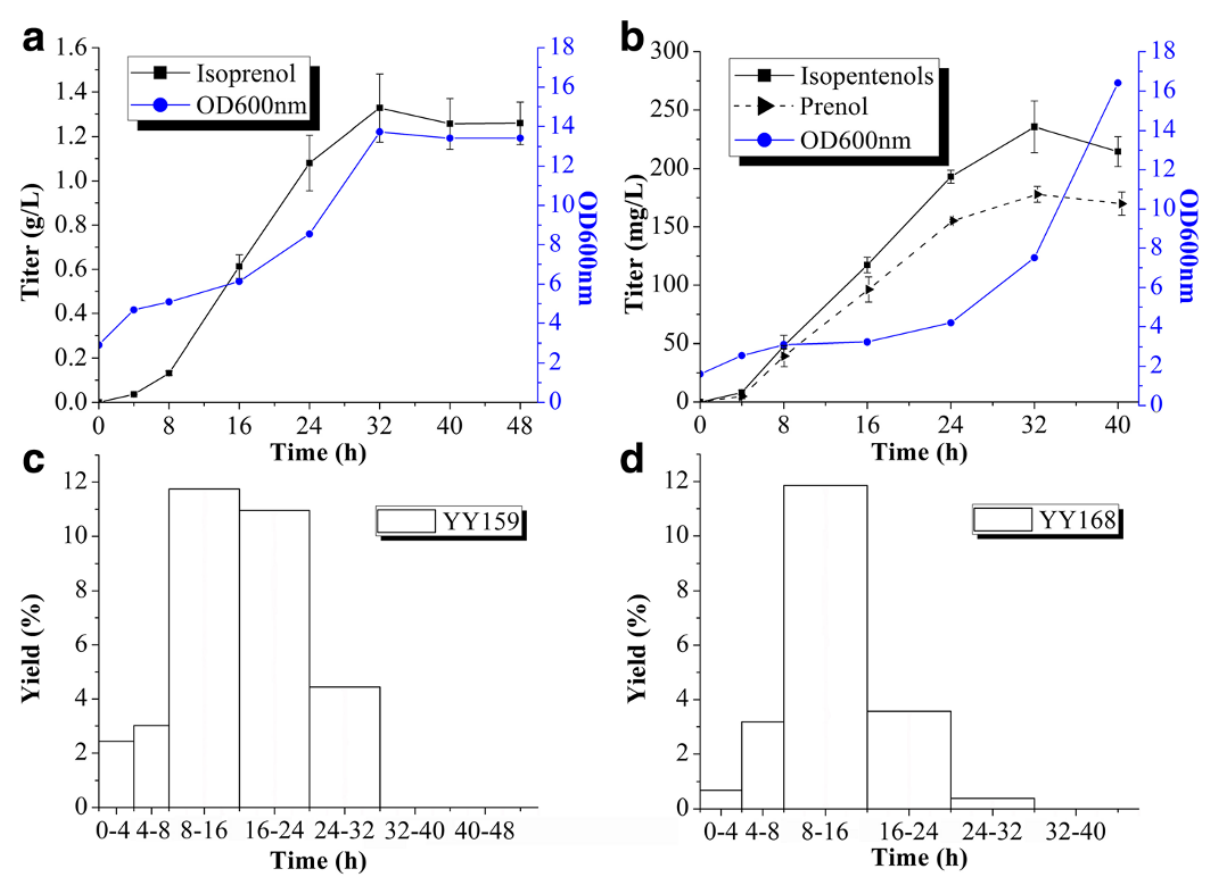

Figure 6 Characterization of isopentenol production of two best-performed strains YY159 and YY168. YY159, expressing pyrophosphatase BsNudF and the whole MVA pathway except IDI1; YY168, expressing pyrophosphatase EcNudF and the whole MVA pathway. YY159 produced a single isoprenol, while YY168 produced a major prenol. $\mathbf{a}$, the isoprenol titer and growth curve of YY159; b, the isopentenol titers and growth curve of YY168; $\mathbf{c}$, the isoprenol yield of YY159; $\mathbf{d}$, the isopentenol yield of YY168.

The achieved over $1.3 \mathrm{~g} / \mathrm{L}$ isoprenol represents an encouraging isopentenol titer. In addition, the isopentenols can be isolated with the well-developed butanol recovery techniques, such as gas stripping. Therefore, this strategy provides the potential and possibility for scale-up production of isopentenols by utilizing the butanol production line without too much reconstruction. Altogether, this

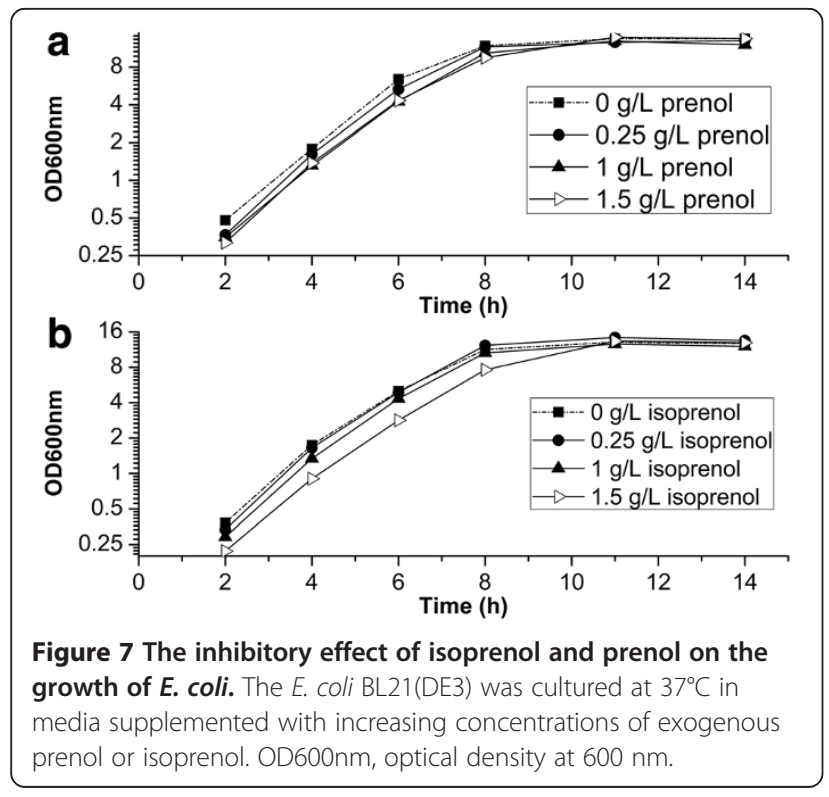

work presents a promising strategy for high-specificity production of two excellent biofuels, isoprenol and prenol.

\section{Methods}

Plasmid construction

The HMGS [Genbank: NM_001182489] was polymerase chain reaction (PCR)-amplified from genomic DNA of $S$. cerevisiae (ATCC 204508) with the primer set HMGS-F and HMGS-R. The PCR product digested with NdeI and XhoI was cloned into pACYCDuet-1 (Novagen, Darmstadt, Germany) cut with the same restriction enzymes, creating pISP211. The tHMGR (a truncated version of $H M G R$ ) was PCR-amplified from genomic DNA of $S$. cerevisiae (ATCC 204508) with the primer set tHMGR-F and tHMGR-R $[7,12]$. The PCR product digested with NcoI and BamHI was cloned into PISP211 cut with the same restriction enzymes, creating pISP212.

The mvaE [Genbank: AF290092] was PCR-amplified from genomic DNA of Enterococcus faecalis (ATCC 700802D-5) with the primer set mvaE-F and mvaE-R. The PCR product digested with NcoI and BamHI was cloned into pACYCDuet-1 cut with the same restriction enzymes, creating pISP213. The mvaS [Genbank: AF290092] was PCR-amplified from genomic DNA of $E$. faecalis (ATCC 700802D-5) with the primer set mvaS-F and mvaS-R. The PCR product digested with SacI and 
Table 1 Bacterial strains and plasmids used in this study

\begin{tabular}{|c|c|c|}
\hline Plasmid or strain & Relevant genotype or description & Reference \\
\hline \multicolumn{3}{|l|}{ Plasmids } \\
\hline pACYCDuet-1 & $\mathrm{P} 15 \mathrm{~A}$ origin; $\mathrm{Cm}^{\mathrm{R}} ; \mathrm{P}_{\mathrm{T} 7}$ & Novagen \\
\hline pTrcHis2B & ColE1 origin; $A m p^{R} ; P_{\text {tre }}$ & Invitrogen \\
\hline pCOLADuet-1 & ColA origin; $\mathrm{Kan}^{\mathrm{R}} ; \mathrm{P}_{\mathrm{T} 7}$ & Novagen \\
\hline pISP212 & $\mathrm{P} 15 \mathrm{~A}$ origin; $\mathrm{Cm}^{\mathrm{R}} ; \mathrm{P}_{\mathrm{T} 7}: . H M G S(\mathrm{Sc})-\mathrm{tHMGR}(\mathrm{Sc})$ & This study \\
\hline plSP214 & P15A origin; $\mathrm{Cm}^{\mathrm{R}} ; \mathrm{P}_{\mathrm{T} 7}:$ mvaS(Ef)-mvaE(Ef) & This study \\
\hline plSP214m & P15A origin; $\mathrm{Cm}^{\mathrm{R}} ; \mathrm{P}_{\mathrm{T} 7: . m v a S A 110 \mathrm{G}-m v a E(E f)}$ & This study \\
\hline pTrcLower & ColE1 origin; $\mathrm{Amp}^{\mathrm{R}}$; $\mathrm{P}_{\mathrm{trc}}:$ ERG12(Sc)-ERG8(Sc)-ERG19(Sc)-IDI1(Sc) & [19] \\
\hline plSP9 & ColE1 origin; $\mathrm{Amp}^{\mathrm{R}}$; $\mathrm{P}_{\mathrm{trc}}:$ ERG12(Sc)-ERG8(Sc)-ERG19(Sc) & This study \\
\hline pYY11 & 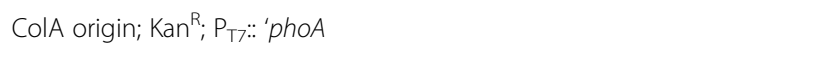 & This study \\
\hline pYY12 & ColA origin; $\mathrm{Kan}^{\mathrm{R}}$; $\mathrm{P}_{\mathrm{T} 7 .:}$ 'DPP1 & This study \\
\hline pYY13 & ColA origin; Kan ${ }^{\mathrm{R}}$; $\mathrm{P}_{\mathrm{T} 7: .:}$ 'LPP1 & This study \\
\hline pYY14 & ColA origin; $\mathrm{Kan}^{\mathrm{R}}$; $\mathrm{P}_{\mathrm{T} 7::}$ phoE & This study \\
\hline pYY15 & ColA origin; Kan ${ }^{\mathrm{R}} ; \mathrm{P}_{\mathrm{T} 7:: B s N u d F}$ & This study \\
\hline pYY16 & ColA origin; Kan ${ }^{R} ; P_{T 7}: E C N u d F$ & This study \\
\hline pYY26 & ColA origin; $\mathrm{Kan}^{\mathrm{R}} ; \mathrm{P}_{\mathrm{T} 7: \text { :NudC }}$ & This study \\
\hline pYY36 & 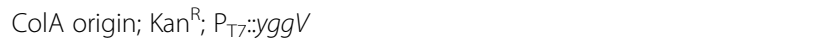 & This study \\
\hline pYY46 & ColA origin; $\mathrm{Kan}^{\mathrm{R}} ; \mathrm{P}_{\mathrm{T} 7}:$ hisl & This study \\
\hline pYY56 & 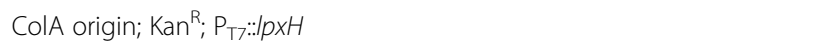 & This study \\
\hline pYY66 & 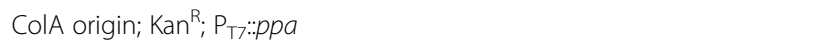 & This study \\
\hline pYY76 & ColA origin; $\operatorname{Kan}^{\mathrm{R}} ; \mathrm{P}_{\mathrm{T} 7: .} \mathrm{cdh}$ & This study \\
\hline \multicolumn{3}{|l|}{ Strains } \\
\hline $\mathrm{DH} 5 \mathrm{a}$ & deo $R$, recA1, endA1, hsdR17(rk, mk $\left.{ }^{+}\right)$, phoA, supE44, $\lambda-$, thi'1, gyrA96, relA1 & Takara \\
\hline BL21(DE3) & E. coli B dcm ompT hsdS(r- $\left.\mathrm{r}_{\mathrm{B}}^{-}\right) \mathrm{gal}$ & Invitrogen \\
\hline W 3110 & $F^{-} \lambda^{-}$rph-1 INV(rrnD, rrnE) & {$[24]$} \\
\hline DNF(DE3) & W3110: $\triangle E C N u d F(D E 3)$ & This study \\
\hline DNFYV(DE3) & W3110: $\Delta E c N u d F \Delta y g g V(D E 3)$ & This study \\
\hline ISP212 & BL21(DE3) harboring pISP212 & This study \\
\hline ISP214 & BL21(DE3) harboring pISP214 & This study \\
\hline ISP214m & BL21(DE3) harboring plSP214m & This study \\
\hline ISP2148 & BL21(DE3) harboring pISP214 and pTrcLower & This study \\
\hline ISP2149 & BL21(DE3) harboring pISP214 and pISP9 & This study \\
\hline YY118 & BL21(DE3) harboring pISP214, pTrcLower and pYY11 & This study \\
\hline YY128 & BL21(DE3) harboring pISP214, pTrcLower and pYY12 & This study \\
\hline YY138 & BL21(DE3) harboring pISP214, pTrcLower and pYY13 & This study \\
\hline YY148 & BL21(DE3) harboring pISP214, pTrcLower and pYY14 & This study \\
\hline YY158 & BL21(DE3) harboring pISP214, pTrcLower and pYY15 & This study \\
\hline YY168 & BL21(DE3) harboring pISP214, pTrcLower and pYY16 & This study \\
\hline YY119 & BL21(DE3) harboring pISP214, pISP9 and pYY11 & This study \\
\hline YY129 & BL21(DE3) harboring pISP214, pISP9 and pYY12 & This study \\
\hline YY139 & BL21(DE3) harboring pISP214, pISP9 and pYY13 & This study \\
\hline YY149 & BL21(DE3) harboring pISP214, pISP9 and pYY14 & This study \\
\hline YY159 & BL21(DE3) harboring pISP214, pISP9 and pYY15 & This study \\
\hline YY169 & BL21(DE3) harboring pISP214, pISP9 and pYY16 & This study \\
\hline
\end{tabular}


Table 1 Bacterial strains and plasmids used in this study (Continued)

\begin{tabular}{lll}
\hline YY159m & BL21(DE3) harboring pISP214m, pISP9 and pYY15 & This study \\
YY168m & BL21(DE3) harboring pISP214m, pTrcLower and pYY16 & This study \\
YY268 & BL21(DE3) harboring pISP214, pTrcLower and pYY26 & This study \\
YY368 & BL21(DE3) harboring pISP214, pTrcLower and pYY36 & This study \\
YY468 & BL21(DE3) harboring pISP214, pTrcLower and pYY46 & This study \\
YY568 & BL21(DE3) harboring pISP214, pTrcLower and pYY56 & This study \\
YY668 & BL21(DE3) harboring pISP214, pTrcLower and pYY66 & This study \\
YY768 & BL21(DE3) harboring pISP214, pTrcLower and pYY76 & This study \\
\hline
\end{tabular}

PstI was cloned into pISP213 cut with the same restriction enzymes, creating pISP214.

The ERG12, ERG8, ERG19 and IDI1 genes from $S$. cerevisiae (ATCC 204508) were cloned into pTrcHis2B (Invitrogen, Carlsbad, CA) using a method of successive hybridization to yield pTrcLower [19].

To delete the IDI1 gene from pTrcLower, a fragment containing ERG19, ERG8 and a part of ERG12 was first PCR-amplified from pTrcLower with the primers IDIKO-F and IDIKO-R, then digested with BamHI and PstI, and finally cloned into the pTrcLower cut with the same restriction enzymes. The resultant recombinant plasmid designated pISP9.

The 'phoA (a leaderless version of phoA [Genbank: NC_000913.2:400971..402386]) was PCR-amplified from genomic DNA of $E$. coli $\mathrm{K} 12$ with the primer set phoAF2 and phoA-R. The PCR product digested with BglII and XhoI was cloned into pCOLADuet-1 (Novagen, Darmstadt, Germany) cut with the same restriction enzymes, creating pYY11.

The 'DPP1 (a leaderless version of DPP1 [Genbank: NM_001180592]) was PCR-amplified from genomic DNA of $S$. cerevisiae (ATCC 204508) with the primer set DPP1-F2 and DPP1-R. The PCR product digested with BglII and Xhol was cloned into pCOLADuet-1 cut with the same restriction enzymes, creating pYY12.

The 'LPP1 (a leaderless version of LPP1 [Genbank: NM_001180811]) was PCR-amplified from genomic DNA of $S$. cerevisiae (ATCC 204508) with the primer set LPP1F2 and LPP1-R. The PCR product digested with BglII and XhoI was cloned into pCOLADuet-1 cut with the same restriction enzymes, creating pYY13.

The phoE [Genbank: AL009126] was PCR-amplified from genomic DNA of Bacillus subtilis (ATCC 23857) with the primer set phoE-F and phoE-R. The PCR product digested with BglII and XhoI was cloned into pCOLADuet-1 cut with the same restriction enzymes, creating pYY14.

The BsNudF [Genbank: AL009126] was PCR-amplified from genomic DNA of B. subtilis (ATCC 23857) with the primer set BsNudF-F and BsNudF-R. The PCR product digested with $\mathrm{NcoI}$ and BamHI was cloned into
pCOLADuet-1 cut with the same restriction enzymes, creating pYY15.

The EcNudF [Genbank: NC_010473.1:3273048..3273677] was PCR-amplified from genomic DNA of E. coli K12 with the primer set EcNudF-F and EcNudF-R. The PCR product digested with $\mathrm{NcoI}$ and BamHI was cloned into pCOLADuet-1 cut with the same restriction enzymes, creating pYY16.

The nudC, yggV, hisl, lpxH, ppa and $c d h$ were PCRamplified from genomic DNA of E. coli K12 with the primer set nudC-F/nudC-R, yggV-F/yggV-R, hisl-F/hisl-R, lpxH-F/lpxH-R, ppa-F/ppa-R and cdh-F/cdh-R, respectively. The PCR products digested with NcoI and BamHI were cloned into pCOLADuet-1 cut with the same restriction enzymes, creating pYY26, pYY36, pYY46, pYY56, pYY66 and pYY76, respectively.

All the plasmids and strains used in this work are listed in Table 1, and the oligonucleotide primers are given in Table 2.

\section{Site-directed mutagenesis}

A method based on the amplification of the entire plasmid using primers that include the desired changes was employed for the site-directed mutagenesis [25]. The Ala-110 of HMG-CoA synthase MvaS was mutated to Gly by replacing a nucleotide $\mathrm{C}$ with $\mathrm{G}$ using the mutant primers A110G-F and A110G-R.

The PrimeSTAR HS DNA polymerase (Takara) was used for PCR. The PCR conditions were as follows: $98^{\circ} \mathrm{C}$ for $30 \mathrm{sec} ; 12 \times\left(98^{\circ} \mathrm{C}\right.$ for $5 \mathrm{sec} ; 63^{\circ} \mathrm{C}$ for $5 \mathrm{sec} ; 72^{\circ} \mathrm{C}$ for $8 \mathrm{~min}) ; 72^{\circ} \mathrm{C}$ for $10 \mathrm{~min}$. The mutant was verified by sequencing (BGI).

\section{Gene knockout and $\lambda$ DE3 lysogenization}

The EcNudF gene of E. coli was knocked out using the one-step inactivation method previously reported [26]. This method includes following steps: amplification of the kanamycin-resistant $\left(\operatorname{Kan}^{\mathrm{R}}\right)$ gene with PCR using pKD4 as template. The PCR product was transformed into the cells by electro-poration after gel purification. Transformants were selected with kanamycin-resistance plate. The mutant was verified by PCR using the test 
Table 2 Primers used in this study

\begin{tabular}{|c|c|}
\hline Name & Sequence $\left(5^{\prime} \rightarrow 3^{\prime}\right)$ \\
\hline HMGS-F & GGAATTCCATATGAAACTCTCAACTAAACTTTG \\
\hline HMGS-R & CACCTCGAGTTATTITITAACATCGTAAGATC \\
\hline tHMGR-F & CATGCCATGGACCAATTGGTGAAAACTGAAG \\
\hline tHMGR-R & CGGGATCCTTAGGATTTAATGCAGGTGACG \\
\hline mvaS-F & $\begin{array}{l}\text { CCAGAGCTCAGGAGGTAAAAAAACATGACAA } \\
\text { TTGGGATTGATAAAATTA }\end{array}$ \\
\hline mvaS-R & CAACTGCAGTTAGTTTCGATAAGAGCGAACG \\
\hline mvaE-F & $\begin{array}{l}\text { CATGCCATGGAGGAGGTAAAAAAACATGAA } \\
\text { AACAGTAGTTATTATTGATGC }\end{array}$ \\
\hline mvaE-R & CGCGGATCCTTATTGTITCTTAAATCATTIAAAATAG \\
\hline IDIKO-F & GTCTITTGGATCCGTTGTTAGCTC \\
\hline IDIKO-R & CAAAACTGCAGTTATTCCTITGGTAGACCAGTC \\
\hline phoA-F2 & CTCAGATCTCCGGACACCAGAAATGCCTGTTC \\
\hline phoA-R & CACCTCGAGTTATTTCAGCCCCAGAGCGGC \\
\hline DPP1-F2 & CTCAGATCTCCAACCGTTCGAACGTCAGTTTIAC \\
\hline DPP1-R & CACCTCGAGTTACATACCTTCATCGGACAAAG \\
\hline LPP1-F2 & CTCAGATCTCGAGATATCCCTGGTACCTAGGG \\
\hline LPP1-R & CACCTCGAGTTACTAAACACTAACCGGTGAAGGAAG \\
\hline phoE-F & CTAGCCATGGGCATGACAGCCGTTTGTTAAGTAAG \\
\hline phoE-R & CAGGGATCCTTATTTGATAAAGCCGGATAAGTG \\
\hline BsNudF-F & CTAGCCATGGGCATGAAATCATTAGAAGAAAAAAC \\
\hline BsNudF-F & CAGGGATCCTCATTITTGTGCTTGGAGCG \\
\hline EcNudF-F & CTAGCCATGGGCATGCTTAAGCCAGACAACCTG \\
\hline EcNudF-F & CAGGGATCCTTATGCCCACTCATIIITAACG \\
\hline nudC-F & CTAGCCATGGATCGTATAATTGAAAAATTAG \\
\hline nudC-R & CAGGGATCCTCACTCATACTCTGCCCGACAC \\
\hline yggV-F & CTAGCCATGGGCATGCAAAAAGTTGTCCTCGCAAC \\
\hline yggV-R & CAGGGATCCTTAACCATTACGTAAAGCGTCC \\
\hline hisl-F & CTAGCCATGGGCATGTTAACAGAACAACAACGTCG \\
\hline hisl-R & CAGGGATCCTCACTGATGCCGTTAACGCAG \\
\hline IpxH-F & CTAGCCATGGCGACACTCTITATTGCAG \\
\hline IpxH-R & CAGGGATCCTTAAAACGGAAAATGAATCAGCTC \\
\hline ppa-F & CTAGCCATGGGCATGAGCTTACTCAACGTCCCTG \\
\hline ppa-R & CAGGGATCCTTATTTATTCTTTGCGCGCTCG \\
\hline cdh-F & CTAGCCATGGGCATGAAAAAAGCGGGTCTTCT \\
\hline cdh-R & CAGGGATCCTTAACGCAAAATCTCACACTG \\
\hline KnudF-F & $\begin{array}{l}\text { TCGCTGAAATTCACATTIAATTCACTATTAGT } \\
\text { GCCAGGACATTAACAATGCATGGGAATTAGCCATGGTCC }\end{array}$ \\
\hline KnudF-R & $\begin{array}{l}\text { TCAGGAAAGTCAGGTGTGTAACGCTTCATTTATGCCCA } \\
\text { CTCATIIITAATGTAGGCTGGAGCTGCTTCG }\end{array}$ \\
\hline KyggV-F & $\begin{array}{l}\text { GCAACAAATCCCGCCAGAAATCGCGGCGTTAATTAATT } \\
\text { AGGTATCCTATGCATGGGAATTAGCCATGGTCC }\end{array}$ \\
\hline KyggV-R & $\begin{array}{l}\text { TGTGAATGTAGAGACTCAGCGGAGGTAATTTAACCATT } \\
\text { ACGTAAAGCGTCTGTAGGCTGGAGCTGCTTCG }\end{array}$ \\
\hline KnudF-vF & ACCCAGAAAGGCTCAGGCCG \\
\hline KnudF-vR & ACAGTITCGCCGGGTGCGTC \\
\hline
\end{tabular}

Table 2 Primers used in this study (Continued)

\begin{tabular}{ll}
\hline KyggV-vF & GACGGCCAGGCCAACAGTCA \\
KyggV-vR & CGGCCCTGAGCGTAAGCCAC \\
A110G-F & CTCTITCGAAATCAAGGAAGGTTGTACGGAGC \\
A110G-R & CCTTCCTTGATTCGAAAGAGCGAGCGAAAG \\
\hline Underlines indicate restriction enzyme sites.
\end{tabular}

Underlines indicate restriction enzyme sites.

primers KOnudF-VF/KOnudF-VR. The kanamycin cassette was removed with the helper plasmid pCP20 that expresses FLP. The E. coli DNF was finally obtained after removing the helper plasmids.

To express target genes cloned in vectors under the control of the T7 promoter, $\lambda \mathrm{DE} 3$ prophage was integrated into the $E$. coli DNF chromosome using the $\lambda \mathrm{DE} 3$ lysogenization kit (Novagen, Darmstadt, Germany) according to the manufacturer's instructions. The obtained strain was designated as E. coli DNF (DE3). The $y g g V$ gene was then deleted with the same method using $E$. coli DNF (DE3) as the starting strain. The resultant double-gene knockout mutant was designated as E. coli DNFYV (DE3).

\section{Bacterial strains, media and growth conditions}

The bacterial strains used in this study are listed in Table 1. E. coli BL21(DE3) (Invitrogen, Carlsbad, CA) was used as the host to overproduce proteins. During strain construction, cultures were grown aerobically at $37^{\circ} \mathrm{C}$ in Luria Broth $(10 \mathrm{~g} / \mathrm{L}$ tryptone, $10 \mathrm{~g} / \mathrm{L} \mathrm{NaCl}$, and $5 \mathrm{~g} / \mathrm{L}$ yeast extract). Kanamycin $(50 \mathrm{mg} / \mathrm{L})$, Ampicillin $(100 \mathrm{mg} / \mathrm{L})$ or chloramphenicol $(34 \mathrm{mg} / \mathrm{L})$ was added if necessary. For initial production experiments in shake flasks, strains were grown in a medium consisted of the following: $7.5 \mathrm{~g} / \mathrm{L}$ $\mathrm{K}_{2} \mathrm{HPO}_{4} \cdot 3 \mathrm{H}_{2} \mathrm{O}, 2.1 \mathrm{~g} / \mathrm{L}$ citric acid monohydrate, $0.3 \mathrm{~g} / \mathrm{L}$ ferric ammonium citrate, $2.92 \mathrm{~g} / \mathrm{L}(\mathrm{NH} 4)_{2} \mathrm{SO}_{4}, 30 \mathrm{~g} / \mathrm{L}$ of glucose, $9 \mathrm{~g} / \mathrm{L}$ beef extract, $4 \mathrm{mM} \mathrm{MgSO}$, trace metals mix (2.86 mg/L $\mathrm{H}_{3} \mathrm{BO}_{3}, 1.81 \mathrm{mg} / \mathrm{L} \quad \mathrm{MnCl}_{2} \cdot 4 \mathrm{H}_{2} \mathrm{O}$, $0.222 \mathrm{mg} / \mathrm{L} \mathrm{ZnSO}_{4} \cdot 7 \mathrm{H}_{2} \mathrm{O}, 0.39 \mathrm{mg} / \mathrm{L} \mathrm{Na}_{2} \mathrm{MoO}_{4} \cdot 2 \mathrm{H}_{2} \mathrm{O}$, $\left.0.079 \mathrm{mg} / \mathrm{L} \mathrm{CuSO}_{4} \cdot 5 \mathrm{H}_{2} \mathrm{O}, 49.4 \mu \mathrm{g} / \mathrm{L} \mathrm{Co}\left(\mathrm{NO}_{3}\right)_{2} \cdot 6 \mathrm{H}_{2} \mathrm{O}\right)$. The residual glucose was measured by the SBA-40D Biosensor equipped with glucose oxidase membrane electrodes (Shandong Academy of Sciences, Jinan, China). Protein production was induced with $0.5 \mathrm{mM}$ isopropyl $\beta$ D-thiogalactoside (IPTG) at $30^{\circ} \mathrm{C}$.

\section{Determination of mevalonate}

Ten milliliter cell free cultures were collected, adjusted to $\mathrm{pH} 2.0$ using hydrochloric acid, and incubated at $45^{\circ} \mathrm{C}$ for 1 hour. Then $5 \mathrm{~g}$ of anhydrous granular sodium sulphate was added to each vial followed by $10 \mathrm{ml}$ of ethyl acetate. The vials were mixed on a vortexer for $5 \mathrm{~min}$ and the phases were allowed to separate naturally. One milliliter supernatant was collected for gas chromatograph (GC) analysis. The separation of mevolonatone lactone was performed using a CP-FFAP CB capillary column 
(25 m $\times 0.25 \mu \mathrm{mm} ; 0.2 \mu \mathrm{m}$ film thickness) purchased from Agilent Technologies (Santa Clara, CA). The oven temperature was initially held at $150^{\circ} \mathrm{C}$ for $1 \mathrm{~min}$, then raised with a gradient of $10^{\circ} \mathrm{C} / \mathrm{min}$ until reaching $250^{\circ} \mathrm{C}$, and finally held at $250^{\circ} \mathrm{C}$ for $10 \mathrm{~min}$. Nitrogen was used as the carrier gas. The injector and detector were held at $250^{\circ} \mathrm{C}$ and $270^{\circ} \mathrm{C}$, respectively.

\section{Analysis of isoprenol and prenol by GC-MS}

Isoprenol and prenol produced by the engineered strains were identified by GC-MS. The system consisted of model 7890A network GC system (Agilent Technologies) and a model 5975C network mass selective detector (Agilent Technologies, Santa Clara, CA). A HP-INNOWAX capillary column $(30 \mathrm{~m} \times 0.25 \mathrm{~mm}$; $0.25 \mu \mathrm{m}$ film thickness; Agilent Technologies) was used, with helium as the carrier gas. The following oven temperature program was carried out: $100^{\circ} \mathrm{C}$ for $1 \mathrm{~min}$, increase of $5^{\circ} \mathrm{C} / \mathrm{min}$ to $100^{\circ} \mathrm{C}$, then programmed from $100^{\circ} \mathrm{C}$ to $200^{\circ} \mathrm{C}$ at $25^{\circ} \mathrm{C} / \mathrm{min}$. The injector was maintained at $250^{\circ} \mathrm{C}$. Alcohol compounds were isolated by ethyl acetate extraction. A $1 \mu \mathrm{l}$ sample was injected in split injection mode with a 20:1 split ratio.

\section{Analysis of isoprenol and prenol by GC-FID}

The produced isoprenol and prenol were quantified by a GC equipped with flame ionization detector (FID). The separation of isoprenol and prenol was performed using a CP-FFAP CB capillary column $(25 \mathrm{~m} \times 0.25 \mathrm{~mm} ; 0.2 \mu \mathrm{m}$ film thickness) purchased from Agilent Technologies (Santa Clara, CA). The oven temperature was initially held at $50^{\circ} \mathrm{C}$ for $1 \mathrm{~min}$, then raised with a gradient of $5^{\circ} \mathrm{C} / \mathrm{min}$ until reaching $100^{\circ} \mathrm{C}$, and finally programmed to $150^{\circ} \mathrm{C}$ at $25^{\circ} \mathrm{C} / \mathrm{min}$. Nitrogen was used as the carrier gas. The injector and detector were held at $250^{\circ} \mathrm{C}$ and $270^{\circ} \mathrm{C}$, respectively. Samples were prepared by ethyl acetate extraction. Isoamyl alcohol was added into the samples as the internal standard before solvent extraction.

\section{Abbreviations}

IPP: Isopentenyl pyrophosphate; DMAPP: Dimethylallyl pyrophosphate; IPTG: Isopropyl $\beta$-D-thiogalactoside; PCR: Polymerase chain reaction; GC: Gas chromatography; FID: Flame ionization detector; GC-MS: Gas

chromatography-mass spectrometry.

\section{Competing interests}

The authors declare that they have no competing interests.

\section{Authors' contributions}

YZ designed the research and prepared the manuscript. MX and WQ helped to revise the manuscript. $Y Z, Q L$ and $L L$ did the lab work, plasmid construction, site-directed mutagenesis, strain cultivation and product detection. JY, HZ, XJ and TC did some work in plasmid construction. WL and XX did some work in product detection. All authors read and approved the final manuscript.

\section{Acknowledgements}

This work was financially supported by the National Natural Science Foundation of China (Grant No. 21202179; Grant No. 21206185), the Knowledge Innovation Program of the Chinese Academy of Sciences (Grant
No. Y112131105), the National High-tech R\&D Program of China (863 Program) (Grant No. SS2013AA050703-2), and the National Science and Technology Development Program for Rural Areas during the 12th Five-Year Plan Period (Grant No. 2012BAD32B06-2).

\section{Author details}

${ }^{1}$ CAS Key Laboratory of Biobased Materials, Qingdao Institute of Bioenergy and Bioprocess Technology, Chinese Academy of Sciences, No.189 Songling Road, Laoshan District, Qingdao 266101, China. ${ }^{2}$ University of Chinese Academy of Sciences, Beijing 100049, China. ${ }^{3}$ College of Food Science, Sichuan Agricultural University, Yaan 625014, China.

Received: 3 February 2013 Accepted: 12 April 2013

Published: 24 April 2013

\section{References}

1. Atsumi S, Hanai T, Liao JC: Non-fermentative pathways for synthesis of branched-chain higher alcohols as biofuels. Nature 2008, 451:86-89.

2. Steen EJ, Kang Y, Bokinsky G, Hu Z, Schirmer A, McClure A, del Cardayre SB, Keasling JD: Microbial production of fatty-acid-derived fuels and chemicals from plant biomass. Nature 2010, 463:559-562.

3. Bond-Watts BB, Bellerose RJ, Chang MCY: Enzyme mechanism as a kinetic control element for designing synthetic biofuel pathways. Nat Chem Biol 2011, 7:222-227.

4. Zheng Y, Li L, Liu Q, Yang J, Cao Y, Jiang X, Zhao G, Xian M: Boosting the free fatty acid synthesis of Escherichia coli by expression of a cytosolic Acinetobacter baylyi thioesterase. Biotechnol Biofuels 2012, 5:76.

5. Koppram R, Nielsen F, Albers E, Lambert A, Wännström S, Welin L, Zacchi G, Olsson L: Simultaneous saccharification and co-fermentation for bioethanol production using corncobs at lab, PDU and demo scales. Biotechnol Biofuels 2013, 6:2.

6. Connor MR, Cann AF, Liao JC: 3-Methyl-1-butanol production in Escherichia coli: random mutagenesis and two-phase fermentation. Appl Microbiol Biotechnol 2010, 86:1155-1164.

7. Martin VJ, Pitera DJ, Withers ST, Newman JD, Keasling JD: Engineering a mevalonate pathway in Escherichia coli for production of terpenoids. Nat Biotechnol 2003, 21:796-802.

8. Ajikumar PK, Xiao WH, Tyo KEJ, Wang Y, Simeon F, Leonard E, Mucha O, Phon TH, Pfeifer B, Stephanopoulos G: Isoprenoid pathway optimization for taxol precursor overproduction in Escherichia coli. Science 2010, 330:70-74.

9. Bonanno JB: Structural genomics of enzymes involved in sterol/ isoprenoid biosynthesis. Proc Natl Acad Sci USA 2001, 98:12896-12901.

10. Wu S, Schalk M, Clark A, Miles RB, Coates R, Chappell J: Redirection of cytosolic or plastidic isoprenoid precursors elevates terpene production in plants. Nat Biotechnol 2006, 24:1441-1447.

11. Agranoff BW, Eggerer H, Henning U, Lynen F: Biosynthesis of terpenes. VII. Isopentenyl pyrophosphate isomerase. J Biol Chem 1960, 235:326-332.

12. Polakowski T, Stahl U, Lang C: Overexpression of a cytosolic hydroxymethylglutaryl-CoA reductase leads to squalene accumulation in yeast. Appl Microbiol Biotechnol 1998, 49:66-71.

13. Tabata K, Hashimoto S: Production of mevalonate by a metabolicallyengineered Escherichia coli. Biotechnol Lett 2004, 26:1487-1491.

14. Wilding El, Brown JR, Bryant AP, Chalker AF, Holmes DJ, Ingraham KA, lordanescu S, So CY, Rosenberg M, Gwynn MN: Identification, evolution, and essentiality of the mevalonate pathway for isopentenyl diphosphate biosynthesis in gram-positive cocci. J Bacteriol 2000, 182:4319-4327.

15. Campos N, Rodríguez-Concepción M, Sauret-Güeto S, Gallego F, Lois LM, Boronat A: Escherichia coli engineered to synthesize isopentenyl diphosphate and dimethylallyl diphosphate from mevalonate : a novel system for the genetic analysis of the 2-C-methyl-D-erythritol 4-phosphate pathway for isoprenoid biosynthesis. Biochem J 2001, 353:59-67.

16. Toth MJ, Huwyler L: Molecular cloning and expression of the CDNAs encoding human and yeast mevalonate pyrophosphate decarboxylase. J Biol Chem 1996, 271:7895-7898.

17. Tsay $Y H$, Robinson GW: Cloning and characterization of $E R G 8$, an essential gene of Saccharomyces cerevisiae that encodes phosphomevalonate kinase. Mol Cell Biol 1991, 11:620-631.

18. Oulmouden A, Karst F: Nucleotide sequence of the ERG12 gene of Saccharomyces cerevisiae encoding mevalonate kinase. Curr Genet 1991, 19:9-14. 
19. Jiang X, Yang J, Zhang H, Zou H, Wang C, Xian M: In vitro assembly of multiple DNA fragments using successive hybridization. PLoS One 2012, 7:e30267.

20. Tokuhiro K, Muramatsu M, Ohto C, Kawaguchi T, Obata S, Muramoto N, Hirai M, Takahashi H, Kondo A, Sakuradani E, Shimizu S: Overproduction of geranylgeraniol by metabolically engineered Saccharomyces cerevisiae. Appl Environ Microbiol 2009, 75:5536-5543.

21. Kim EE, Wyckoff HW: Reaction mechanism of alkaline phosphatase based on crystal structures: Two-metal ion catalysis. J Mol Biol 1991, 218:449-464.

22. Withers ST, Gottlieb SS, Lieu B, Newman JD, Keasling JD: Identification of isopentenol biosynthetic genes from Bacillus subtilis by a screening method based on isoprenoid precursor toxicity. Appl Environ Microb 2007, 73:6277-6283.

23. Steussy CN, Robison AD, Tetrick AM, Knight JT, Rodwell WW, Stauffacher CV, Sutherlin AL: A structural limitation on enzyme activity: the case of HMGCoA synthase. Biochemistry 2006, 45:14407-14414.

24. Hayashi K, Morooka N, Yamamoto Y, Fujita K, Isono K, Choi S, Ohtsubo E, Baba T, Wanner BL, Mori H, Horiuchi T: Highly accurate genome sequences of Escherichia coli K-12 strains MG1655 and W3110. Mol Syst Biol 2006, 2:2006. 0007.

25. Hemsley A, Arnheim N, Toney MD, Cortopassi G, Galas DJ: A simple method for site-directed mutagenesis using the polymerase chain reaction. Nucleic Acids Res 1989, 17:6545-6551.

26. Datsenko KA, Wanner BL: One-step inactivation of chromosomal genes in Escherichia coli K-12 using PCR products. Proc Natl Acad Sci U S A 2000, 97:6640-6645.

doi:10.1186/1754-6834-6-57

Cite this article as: Zheng et al: Metabolic engineering of Escherichia coli for high-specificity production of isoprenol and prenol as next generation of biofuels. Biotechnology for Biofuels 2013 6:57.

\section{Submit your next manuscript to BioMed Central and take full advantage of:}

- Convenient online submission

- Thorough peer review

- No space constraints or color figure charges

- Immediate publication on acceptance

- Inclusion in PubMed, CAS, Scopus and Google Scholar

- Research which is freely available for redistribution 